\title{
Correlation Transmission Line Matrix (CTLM) Modeling of Stochastic Electromagnetic Fields
}

\author{
Johannes A. Russer*, Andreas Cangellaris ${ }^{\dagger}$, and Peter Russer* \\ *Institute for Nanoelectronics, Technische Universität München, Germany, e-mail: jrusser@tum. de, \\ $\dagger$ University of Illinois at Urbana-Champaign, Urbana, IL 61801 cangella@illinois .edu
}

\begin{abstract}
In this work we introduce the Correlation Transmission Line Matrix (CTLM) method for time-domain computation of the auto- and cross correlation functions (ACFs and CCFs) of stationary stochastic electromagnetic fields. These ACFs and CCFs are computed from the Johns matrices, i.e. the discretetime TLM Green's functions and are directly related to the EMI power spectra.
\end{abstract}

\section{INTRODUCTION}

Future computing and communication systems will exhibit high data bandwidth demands of up to $100 \mathrm{~Gb} / \mathrm{s}$ [1]. On the one hand, switching operations in such broadband circuits will generate radiated electromagnetic interference (EMI), and on the other hand, due to the high bandwidth and the low power levels these circuits also will be very sensitive to EMI. Therefore coexistence of systems with ubiquitous electromagnetic noise and reliable operation requires a careful circuit and system design accounting for signal integrity (SI).

Advanced computer aided design techniques accounting for electromagnetic compatibility (EMC) and SI will be crucial for reducing time-to-market. Integrated circuits are main sources of radiated EMI in electronic circuits and systems. Therefore the EMI radiated from the integrated circuits has to be assessed [2]-[4]. Also interconnects in printed circuit boards can be sources of radiated EMI [5].

In a stochastic EM field numerical amplitudes cannot be specified for the field values. A complete description of the stochastic EM field with Gaussian amplitude probability distribution can be given by specifying all auto correlation functions of the field amplitudes and the cross correlation functions of each pair of field variables [6]. The auto and cross correlation functions of the field variables in the observation points $O P_{1}$ and $O P_{2}$ in Fig. 1 can be computed if the auto and cross correlation functions of the sources $S_{1} \ldots S_{5}$ are known.

The transmission line matrix (TLM) method is an efficient time- and space discrete numerical method for modeling of complex electromagnetic structures [7], [8]. In this work, we introduce the Correlation Transmission Line Matrix (CTLM) method for time-domain computation of the auto- and cross correlation functions (ACFs and CCFs) of stationary stochastic electromagnetic fields. These ACFs and CCFs are computed from the Johns matrices, i.e. the discrete-time TLM Green's functions and are directly related to the EMI power spectra. By convolution of the Johns matrices second order discrete Green's functions - relating the correlation functions of pairs

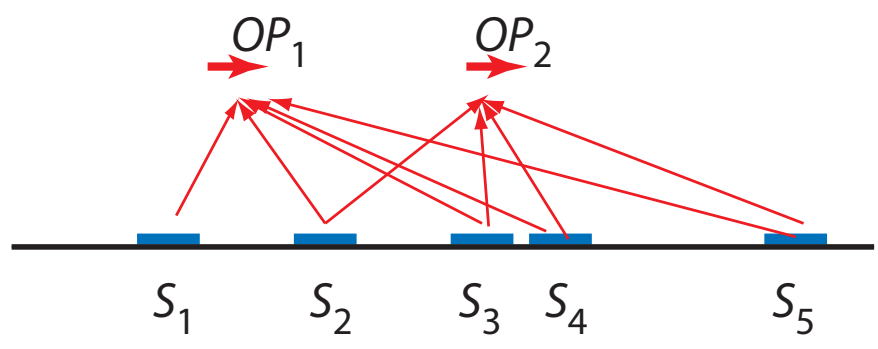

Fig. 1. Stochastic sources $S_{1} \ldots S_{5}$ and observation points $O P_{1}$ and $O P_{2}$.

of observation points to the correlation functions of all pairs of source points - are introduced.

\section{STOCHASTIC EM FIELDS}

The stochastic electric field with the time-windowed amplitude spectrum $\boldsymbol{E}_{T}\left(\boldsymbol{x}_{a}, \omega\right)$ is represented by the dyadic [6]

$$
\underline{\underline{\Gamma}}_{E}\left(\boldsymbol{x}_{a}, \boldsymbol{x}_{b}, \omega\right)=\lim _{T \rightarrow \infty} \frac{1}{2 T}\left\langle\boldsymbol{E}_{T}\left(\boldsymbol{x}_{a}, \omega\right) \boldsymbol{E}_{T}^{\dagger}\left(\boldsymbol{x}_{b}, \omega\right)\right\rangle,
$$

where the subscript ${ }_{T}$ denotes the amplitude spectrum of the field, time-windowed by a rectangular window covering the time interval $[-T, T]$, and the excitation current density with the time-windowed amplitude spectrum $\boldsymbol{J}_{T}\left(\boldsymbol{x}_{a}, \omega\right)$ is represented by the dyadic

$$
\underline{\underline{\Gamma}}_{J}\left(\boldsymbol{x}_{a}, \boldsymbol{x}_{b}, \omega\right)=\lim _{T \rightarrow \infty} \frac{1}{2 T}\left\langle\boldsymbol{J}_{T}\left(\boldsymbol{x}_{a}, \omega\right) \boldsymbol{J}_{T}^{\dagger}\left(\boldsymbol{x}_{b}, \omega\right)\right\rangle .
$$

The excited electric field $\boldsymbol{E}(\boldsymbol{x}, \omega)$ is related to the source current distribution $\boldsymbol{J}\left(\boldsymbol{x}^{\prime}, \omega\right)$ by

$$
\boldsymbol{E}(\boldsymbol{x}, \omega)=\int_{V} \boldsymbol{G}_{E J}\left(\boldsymbol{x}-\boldsymbol{x}^{\prime}, \omega\right) \boldsymbol{J}\left(\boldsymbol{x}^{\prime}, \omega\right) d^{3} x^{\prime},
$$

where $\boldsymbol{G}_{E J}\left(\boldsymbol{x}-\boldsymbol{x}^{\prime}, \omega\right)$ is the Green's dyadic relating the excited electric field $\boldsymbol{E}(\boldsymbol{x}, \omega)$ to $\boldsymbol{J}(\boldsymbol{x}, \omega)$ and the integration is extended over the whole volume $V$ where $\boldsymbol{J}(\boldsymbol{x}, \omega)$ is nonvanishing [6], [9]. From (3), (2), and (1) we obtain

$$
\begin{array}{r}
\underline{\Gamma}_{E}\left(\boldsymbol{x}_{a}, \boldsymbol{x}_{b}, \omega\right)=\iint_{V} \boldsymbol{G}_{E J}\left(\boldsymbol{x}_{a}-\boldsymbol{x}_{a}^{\prime}\right) \\
\times \underline{\underline{\Gamma}}_{J}\left(\boldsymbol{x}_{a}^{\prime}, \boldsymbol{x}_{b}^{\prime}, \omega\right) \boldsymbol{G}_{E J}^{\dagger}\left(\boldsymbol{x}_{b}-\boldsymbol{x}_{b}^{\prime}\right) d^{3} x_{a}^{\prime} d^{3} x_{b}^{\prime} .
\end{array}
$$

Cite as: Russer, J.A.; Cangellaris, A.; Russer, P., 


\section{Digital Signal PRocessing}

\section{A. Time-Discrete Signals}

The discrete-time signal function $s[n]$ is related to the continuous-time signal function $s(t)$ of length $T_{0}$ by

$$
s[n]=s(n \Delta t), \quad \text { with } T_{0}=N \Delta t,
$$

where $\Delta t$ is the sampling interval. If a real-valued discrete time sequence $x_{j}[n]$ is applied to a linear time-invariant system with the impulse response $h_{i j}[n]$, the output sequence $y_{i}[n]$ is obtained by discrete convolution of the input signal $x_{j}[n]$ with the impulse response $h_{i j}[n]$ as [10, p. 64]

$$
y_{i}[n]=h_{i j}[n] * x_{j}[n] \equiv \sum_{m=-\infty}^{\infty} h_{i j}[n-m] x_{j}[m],
$$

where the symbol $*$ denotes the convolution operation.

\section{B. Discrete-Time Correlation Functions}

The discrete-time correlation function $c_{i j}[n, n+m]$ of two real-valued discrete time sequences $x_{i}[n]$ and $x_{j}[n]$ is defined as $[10$, p. 65]

$$
c_{i j}^{x}[n, n+m]=\left\langle x_{i}[n] x_{j}[n+m]\right\rangle,
$$

where $\langle\cdot\rangle$ denotes the ensemble average. If $s_{i}[n]$ and $s_{j}[n]$ are stationary ergodic processes, $c_{i j}[n, n+m]$ is independent from $n$ and the ensemble average is identical with the time average and we can write

$$
\begin{aligned}
c_{i j}^{x}[m] & =\left\langle x_{i}[n] x_{j}[n+m]\right\rangle \\
& =\lim _{N \rightarrow \infty} \frac{1}{2 N+1} \sum_{n=-N}^{N} x_{1}[n] x_{2}[n+m] .
\end{aligned}
$$

From (6) and (7) we obtain

$$
\begin{aligned}
& \left\langle y_{p}[n] y_{q}[n+m]\right\rangle= \\
& =\sum_{k=-\infty}^{\infty} \sum_{l=-\infty}^{\infty} h_{p r}[k]\left\langle x_{r}[n-k] x_{s}[n+m-l]\right\rangle h_{q s}[l] \\
& c_{p q}^{y}[m]=\sum_{k=-\infty}^{\infty} \sum_{l=-\infty}^{\infty} h_{p r}[k] c_{r s}^{x}[m+k-l] h_{q s}[l]
\end{aligned}
$$

We define the correlation transfer function

$$
k_{p q, r s}^{y}[l]=\sum_{k=-\infty}^{\infty} h_{p r}[k] h_{q s}[l+k]
$$

and can write (10) as

$$
c_{p q}^{y}[m]=\sum_{l=-\infty}^{\infty} k_{p q, r s}[l] c_{r s}^{x}[m-l]
$$

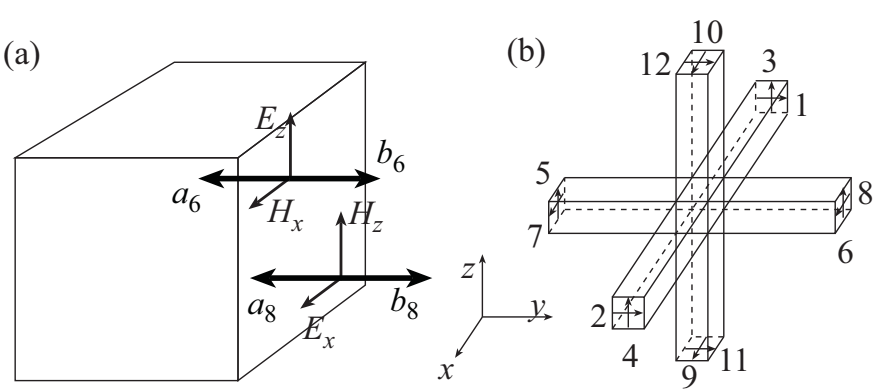

Fig. 2. Schematic of the TLM cell: a) Space cell with samples of the tangential electric and magnetic field values and wave pulse amplitudes, b) TLM node.

\section{TLM - A Discrete Scheme of Electromagnetism}

In the TLM-method, the electromagnetic field is modeled by wave pulses propagating on a Cartesian mesh of transmission lines [11]-[13]. In 3D-TLM with symmetric condensed node, the mesh node is modeled by a twelve-port with the scattering matrix. Figure 2a) exemplifies the assignment of incident and scattered wave amplitudes to the electromagnetic field amplitudes, and Fig. 2b) shows the schematic representation of a symmetric condensed TLM node. To account for the two transverse polarizations, a pair of transmission lines is assigned to every branch of the mesh. In a compact formulation of the TLM scheme we summarize all $12 N$ incident wave pulses in the vector a $[k]$ and all $12 N$ scattered wave pulses in the vector $\mathfrak{b}[k]$. The argument $k$ enumerates the discrete time step. We can formulate the TLM scheme in the compact Hilbert space notation [14]-[16]:

$$
\begin{aligned}
\mathfrak{b}[k+1] & =\boldsymbol{S} \mathrm{a}[k], \\
\mathrm{a}[k] & =\boldsymbol{\Gamma} \mathrm{b}[k],
\end{aligned}
$$

where the scattering matrix $S$ describes the instantaneous scattering of the wave pulses in the TLM node and $\boldsymbol{\Gamma}$ describes the connection of the TLM nodes with the adjacent TLM nodes. The TLM scheme as formulated in (13a) and (13b) is a compact representation of discrete electrodynamics.

\section{DisCRETE TLM GREEN's FunCTIONS}

The response to a wave pulse incident on the boundary of a certain spatial domain may be represented by discrete TLM Green's functions. On the other hand, the response to a localized electromagnetic excitation at the boundary of a certain spatial domain may be calculated directly from Maxwell's equations and be represented by analytic TLM Green's functions. For low frequencies and small wave numbers, the analytic TLM Green's functions coincide with the discrete TLM Green's functions [14], [17]-[19]. Applying the analytic TLM Green's functions in the absorbing boundary condition at the boundary to the open half-space reduces the computational effort considerably when compared with the application of the discrete TLM Green's functions [20]. 


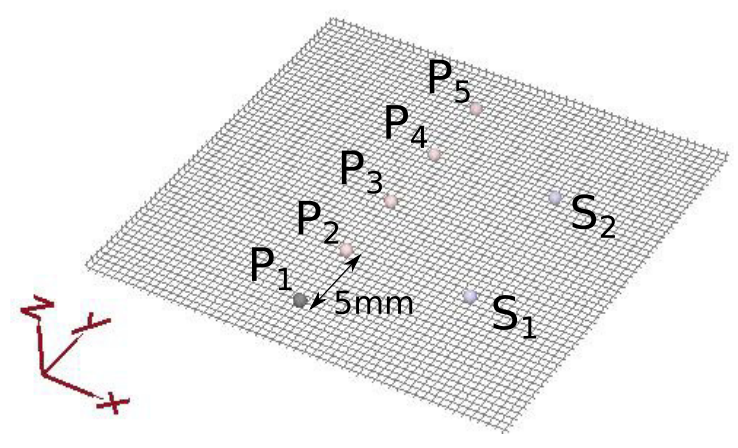

Fig. 3. Arrangement of source and observation points.

The discrete Green's function for TLM can be written as $\mathrm{G}\left[n_{i}, k ; n_{j}, k^{\prime}\right]$ and relates the wave pulses $a\left[n_{i}, k^{\prime}\right]$ incident on boundary port $n_{i}$ and time $k^{\prime}$ to the wave pulses $b\left[n_{j}, k\right]$ scattered from boundary port $n_{j}$ and time $k$ [18], [21]. We can write

$$
b_{i}[k]=\sum_{n_{j} \in B} \sum_{k^{\prime}=-\infty}^{\infty} G_{i, j}\left[k-k^{\prime}\right] a_{j}\left[k^{\prime}\right],
$$

where $B$ is a set $\left\{n_{1}, n_{2}, \ldots n_{N}\right\}$ of $N$ boundary nodes. For stationary stochastic electromagnetic fields we can introduce the following auto- and cross correlation functions of the wave amplitudes:

$$
\begin{aligned}
c_{i j}^{a}[m] & =\left\langle\boldsymbol{a}_{i}[n] \boldsymbol{a}_{j}[n+m]\right\rangle, \\
c_{i j}^{b}[m] & =\left\langle\boldsymbol{b}_{i}[n] \boldsymbol{b}_{j}[n+m]\right\rangle .
\end{aligned}
$$

We introduce the Correlation Green's Function (CGF) $K_{i j ; p q}[k]$ for the TLM wave amplitude correlation functions

$$
K_{i j ; p q}[k]=\sum_{l=-\infty}^{\infty} G_{i, p}[l] G_{j, q}[l+k] .
$$

With (15a), (15b), and (16), we obtain in analogy to (10) the relation

$$
c_{i j}^{b}[m]=\sum_{n_{r}, n_{s} \in B} \sum_{l=-\infty}^{\infty} K_{i j ; r s}[l] c_{r s}^{a}[m-l],
$$

relating the auto- and cross correlation functions $c_{i j}^{b}[m]$ of the wave amplitudes scattered from the boundary to the auto- and cross correlation functions $C_{r s}^{a}[m]$ incident to the boundary.

\section{Numerical EXAMPLE}

In this numerical two-dimensional example, correlation data according to (17) are obtained for five observation points receiving EMI from two sources. This arrangement is depictured in Fig. 3. Impulse responses at the observation points $P_{1}, \cdots, P_{5}$ are computed for excitation at the source points $S_{1}$ and $S_{2}$ using the TLM-based MEFiSTo electromagnetic full wave solver. The impulse excitation is band limited to avoid spurious solutions. Three cases are considered: correlated inphase, correlated antiphase, and uncorrelated sources. The computed autocorrelation functions at the observation points,

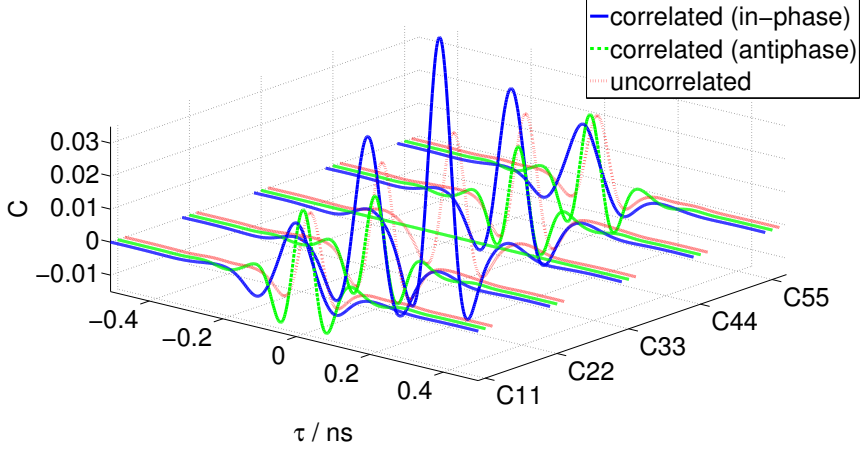

Fig. 4. Time domain autocorrelations of the observation points for a twosource excitation with correlated in-phase, correlated antiphase, and uncorrelated sources.

also a measure for the power density at the respective points, are graphed in Fig. 4. At the central point $P_{3}$, we obtain a power maximum for correlated in-phase sources and zero power for correlated antiphase sources, and pronounced directivity patterns for either case. For the uncorrelated case, this pattern is vanishing. Arbitrary degrees of source correlations may be considered with this methodology. The information obtained in this manner for the propagation of the correlation information are available over a broad bandwidth.

\section{CONCLUSION}

In this work we introduced and applied the Correlation Transmission Line Matrix (CTLM) method for direct computation of the auto and cross correlation functions of stationary stochastic electromagnetic fields. We used Correlation Green's Functions (CGFs) to compute these correlations and gave a numerical example to demonstrate this method. The computation is time-efficient since the correlation depends only on the time delay of the correlation function and not on the time.

\section{ACKNOWLEDGMENT}

This work was supported by the German Research Foundation (DFG) under grant no. Ru 314/45 and by the European Union's Horizon 2020 research and innovation programme under grant no. 664828 (NEMF21).

\section{REFERENCES}

[1] T.-L. Wu, F. Buesink, and F. Canavero, "Overview of signal integrity and EMC design technologies on PCB: fundamentals and latest progress," IEEE Trans. Electromagn. Compat., vol. 55, no. 4, pp. 624-638, 2013.

[2] J. Rollin, G. Areari, and L. Roy, "EMC performance of IC packages," in 1999 IEEE International Symposium on Electromagnetic Compatibility, vol. 1, 1999, pp. 44-46.

[3] M. Bencivinni, F. Camarda, D. Capriglione, A. Chiariello, G. Fusillo, A. Girardi, R. Izzi, A. Maffucci, and I. Martines, "Characterization and modeling of the electromagnetic behavior of ICs and packages," in 2011 Int. Conf. on Electromagn. in Adv. Appl. (ICEAA), 2011, pp. 1368-1371.

[4] J. A. Russer, N. Uddin, A. S. Awny, A. Thiede, and P. Russer, "Nearfield measurement of stochastic electromagnetic fields," Electromagnetic Compatibility Magazine, IEEE, vol. 4, no. 3, pp. 79-85, 2015.

[5] R. Achar, "High-speed interconnect modeling," IEEE Microwave Magazine, vol. 12, no. 5, pp. 61-74, Aug. 2011.

[6] J. A. Russer and P. Russer, "Modeling of noisy EM field propagation using correlation information," IEEE Trans. Microw. Theory Techn. vol. 63, no. 1 , pp. $76-89$, Jan. 2015. 
[7] P. Russer and J. Russer, "Transmission line matrix (TLM) and network methods applied to electromagnetic field computation," in Microwave Symposium Digest (MTT), 2011 IEEE MTT-S International. IEEE, Jun. 2011, pp. 1-4.

[8] P. Russer and J. A. Russer, "Some remarks on the transmission line matrix (TLM) method and its application to transient EM fields and to EMC problems," in Computational Electromagnetics-Retrospective and Outlook, I. Ahmed and Z. D. Chen, Eds. Heidelberg: Springer, August 28 2014, pp. 29-56.

[9] J. A. Russer, F. Mukhtar, O. Filonik, G. Scarpa, and P. Russer, "Modelling of noisy EM field propagation using correlation information of sampled data," in IEEE Int. Conf. on Numerical Electromagnetical Modeling and Optimization NEMO2014, Pavia, Italia, May 14-16 2014.

[10] A. V. Oppenheim and R. W. Schafer, Discrete-Time Signal Processing, 2nd ed., ser. Signal Processing Series. (Prentice-Hall, 1989.

[11] P. Johns and R. Beurle, "Numerical solution of 2-dimensional scattering problems using a transmission-line matrix," Proc. IEE, no. 9, vol. 118 , pp. 1203-1208, Sep. 1971.

[12] W. Hoefer, "The transmission line matrix method-theory and applications," Microwave Theory and Techniques, IEEE Transactions on, vol. 33, no. 10, pp. 882-893, Oct. 1985.

[13] _ "The transmission line matrix (TLM) method," in Numerical Techniques for Microwave and Millimeter Wave Passive Structures, T. Itoh, Ed. New York: J. Wiley, 1989, pp. 496-591.

[14] P. Russer and M. Krumpholz, "The Hilbert space formulation of the TLM method," Int. J. Numerical Modelling: Electronic Networks, Devices and Fields, vol. 6, no. 1, pp. 29-45, Feb. 1993.

[15] M. Krumpholz and P. Russer, "A field theoretical derivation of TLM," IEEE Trans. Microw. Theory Techn., vol. 42, no. 9, pp. 1660-1668, 1994

[16] P. Russer, Electromagnetics, Microwave Circuit and Antenna Design for Communications Engineering, 2nd ed. Boston: Artech House, 2006.

[17] M. Krumpholz and P. Russer, "Discrete time-domain Green's functions for three-dimensional TLM modelling of the radiating boundary conditions," in Proc. 9th Annual Review of Progress in Applied Computational Electromagnetics ACES, Monterey, (Monterey, CA), Mar. 1993, pp. 458466.

[18] M. Krumpholz, B. Bader, and P. Russer, "On the theory of discrete TLM Green's functions in three-dimensional TLM," IEEE Trans. Microw. Theory Techn., vol. 43, no. 7, pp. 1472-1483, 1995.

[19] P. Russer, "The transmission line matrix method," in New Trends and Concepts im Microwave Theory and Technics, H. Baudrand, Ed Trivandrum, India: Research Signpost, 2003, pp. 41-82.

[20] J. A. Russer, Y. Kuznetsov, and P. Russer, "Discrete-time network and state equation methods applied to computational electromagnetics," Mikrotalasna Revija (Microwave Review), pp. 2-14, July 2010.

[21] W. Hoefer, "The discrete time domain Green's function or Johns matrix - a new powerful concept in transmission line modelling," International Journal of Numerical Modelling: Electronic Networks, Devices and Fields, vol. 2, pp. 215-225, 1989. 